\section{Have We Lost the War on Obesity?}

The current issue of the Journal contains a number of manuscripts that are directly relevant to the ongoing obesity pandemic and the public health and policy efforts to address it. One in four Canadian adults and close to 10\% of 6 to 17 year-old children are obese. These prevalences have doubled since the early 1980 s and are much higher in First Nations. Excess weight accounts for 5 to 7 billion dollars in economic costs each year and is a major cause of severe chronic diseases, including diabetes, cardiovascular diseases, several cancers, musculoskeletal diseases and many more.

Many of us have witnessed the unfolding epidemic during our careers in public health. As a society, we Canadians have to a large extent stood by as our collective BMI increased. Sadly, standing by seems to be what we continue to do. Tremblay, in his useful summary of national initiatives against obesity (pg. 164), reminds us that only $9 \%$ of boys and $4 \%$ of girls meet national recommendations on physical activity. This is not surprising when considering the report by Stone and colleagues (pg. 170), who evaluated the Ontario physical activity policy in 16 Toronto schools and found that less than $50 \%$ of children were provided with physical activity opportunities as recommended by the policy and that no children achieved the recommended moderate to vigorous activity levels. Although policies that are actually implemented may be an important means of supporting physical activity, Spence and colleagues (pg.175) convincingly argue that the federal non-refundable tax credit to promote physical activity in children misses the most important target group of low-income families and that it should be replaced by a refundable tax credit.

Physical activity and energy expenditure are one side of the equation. The other is energy intake, and two papers address this topic. Using data from the Canadian Community Health Survey, Nikpartow and colleagues (pg. 178) confirm the growing evidence linking consumption of sugar-sweetened beverages and the risk of excess weight. They report an odds ratio of 2 between a fruit drink pattern of beverage intake and being overweight or obese, but only in Canadian women. Finally, in a report having important policy implications, Williams et al. (pg. 183) report that Nova Scotia households receiving Income Assistance could not afford to purchase a basic nutritious food basket.

These papers collectively show the importance of evidence to support decision-making for obesity prevention and control in Canada. They also underscore the tremendous efforts needed in research, policy and programs to defeat excess weight gain before it defeats us.

\section{Gilles Paradis}

Scientific Editor

\section{Avons-nous perdu la guerre contre l'obésité?}

Le présent numéro de la Revue contient plusieurs manuscrits qui touchent directement à la pandémie d'obésité en cours et aux efforts des décideurs et des praticiens de la santé publique pour y remédier. Au Canada, un adulte sur quatre et près de $10 \%$ des enfants de 6 à 17 ans sont obèses. Ces chiffres de prévalence ont doublé depuis le début des années 1980 et sont beaucoup plus élevés chez les membres des Premières Nations. La surcharge pondérale, qui engendre de 5 à 7 milliards de dollars de coûts économiques par année, est une cause importante de maladies chroniques graves, dont le diabète, les maladies cardiovasculaires, plusieurs cancers, les maladies musculosquelettiques et bien d'autres.

Beaucoup d'entre nous avons été témoins de la déferlante de l'épidémie au cours de notre carrière en santé publique. En tant que société, nous les Canadiens et les Canadiennes avons dans une large mesure fait de la figuration pendant que notre IMC collectif augmentait. Malheureusement, il semble que nous n'avons toujours pas bougé. Tremblay, dans son utile synthèse des initiatives nationales contre l'obésité (p. 164), nous rappelle qu'à peine $9 \%$ des garçons et $4 \%$ des filles respectent les recommandations nationales en matière d'activité physique. Ce n'est pas étonnant lorsqu'on lit le rapport de Stone et coll. (p. 170), qui évaluent l'application de la politique ontarienne sur l'activité physique dans 16 écoles de Toronto et constatent que moins de $50 \%$ des élèves ont droit aux périodes d'activité physique recommandées par la politique, et qu'aucun élève n'atteint le niveau d'activité modérée à vigoureuse recommandé. En autant qu'on les applique, les politiques peuvent être un excellent moyen d'appuyer l'activité physique, mais Spence et coll. (p. 175) soutiennent de façon convaincante que le crédit d'impôt fédéral non remboursable pour promouvoir l'activité physique chez les enfants rate le groupe cible le plus important, celui des familles à faible revenu, et qu'il faudrait le remplacer par un crédit d'impôt remboursable.

L'activité physique et la force dépensée sont un élément de l'équation. L'autre est l'apport énergétique, et deux articles abordent ce sujet. À l'aide des données de l'Enquête sur la santé dans les collectivités canadiennes, Nikpartow et coll. (p. 178) confirment les preuves croissantes qui établissent un lien entre la consommation de boissons édulcorées au sucre et le risque de surcharge pondérale. Ils font état d'un rapport de cotes de 2 entre un profil de consommation de boissons aux fruits et le fait d'être en surpoids ou obèse au Canada, mais seulement chez les femmes. Enfin, dans un rapport qui aura d'importantes répercussions stratégiques, Williams et coll. (p. 183) signalent que les ménages de la Nouvelle-Écosse bénéficiaires de l'aide au revenu n'ont pas les moyens d'acheter un panier d'aliments nutritifs de base.

Ensemble, ces articles montrent l'importance des données probantes pour appuyer la prise de décisions de prévention et de contrôle de l'obésité au Canada. Ils soulignent aussi les efforts immenses qu'il reste à faire, sur les fronts de la recherche, des politiques et des programmes, pour vaincre la prise de poids excessive avant qu'elle ne nous mette en échec.

Le rédacteur scientifique, Gilles Paradis 